\title{
MULTIPLIERS FOR SEMIGROUPS
}

\author{
by G. BLOWER
}

(Received 2nd April 1993)

Let $L$ be a positive invertible self-adjoint operator in $L^{2}(X ; \mathbf{C})$. Using transference methods for locally bounded groups of operators we obtain multipliers for the group of complex powers $L^{i t}$ on vector-valued Lebesgue spaces. Using a Mellin inversion formula, we derive a sufficient condition for a function to be a multiplier of the semigroup $e^{-t L}$ on $L^{p}(X ; E)$ where $E$ is a $U M D$ Banach space and $1<p<\infty$.

1991 Mathematics subject classification: 42A45, 47D03, 46E50.

\section{Introduction}

In this paper we are concerned with multipliers for semigroups generated by a Laplace type operator. Let $X$ be a locally compact manifold and $\mu$ a positive $\sigma$-finite Radon measure supported on $X$. Let $L$ be a densely defined positive self-adjoint operator in $L^{2}(X ; \mathbf{C})$. By the spectral theorem there is a resolution of the identity $P(\mathrm{~d} \lambda)$ consisting of an increasing family of orthogonal projections $P[0, \lambda]$. We suppose that the projection $P_{\{0\}}$ corresponding to the singleton $\{0\}$ is zero. We can define a family of operators

$$
\exp (-t L)=\int_{0}^{\infty} \exp (-t \lambda) P(\mathrm{~d} \lambda) \quad(t>0)
$$

which by Theorems 22.3.1 and 12.3.1 of [4] forms a $C_{0}$ contraction semigroup on $L^{2}(X ; \mathrm{C})$ with generator $(-L)$.

In many examples of interest, $\exp (-t L)$ also defines a semigroup on $L^{p}(X ; \mathbf{C})$. For technical reasons we suppose that there is a common core $\mathscr{C}$ so that $L$ may be unambiguously defined on all the $L^{p}$ spaces. We state the technical hypotheses here and provide examples in Section 5.

Hypotheses 1.1. (i) Let $\mathscr{C}$ be the space of functions

$$
\mathscr{C}=\left\{f \in \bigcap_{1<p<\infty} L^{p}(X ; \mathrm{C}): \operatorname{support}((P f, f)(\mathrm{d} \lambda)) \subset\left(\varepsilon, \varepsilon^{-1}\right), \quad \varepsilon>0\right\}
$$

We assume that $\mathscr{C}$ is a dense linear subspace of $L^{p}(X ; \mathrm{C})$ for $1<p<\infty$.

(ii) The space $\mathscr{C}$ is a core for $L$. We suppose that $L$ is closable on $L^{p}(X ; \mathbf{C})$ for $1<p<\infty$ and its graph closure is equal to the closure of its restriction to $\mathscr{C}$. 
(iii) We assume that $L$ generates a $C_{0}$ semigroup on $L^{p}(X ; \mathrm{C})$ for $1<p<\infty$.

(iv) We let $E$ be some Banach space and for $1 \leqq p<\infty$ introduce the BochnerLebesgue space $L^{p}(X ; E)$ of strongly measurable $E$-valued functions with $\|f(x)\|_{E}^{p}$ $\mu$-integrable as in $\left[4\right.$, p. 78]. Suppose that $e^{-t L} \otimes I$, defined initially on $\mathscr{C} \otimes E$, may be extended to a $C_{0}$ semigroup, also denoted $\exp (-t L)$, on $L^{p}(X ; E)$ for $1<p<\infty$. We assume that $\mathscr{C} \otimes E$ forms a comon core for $L \otimes I$ in the $L^{p}(X ; E)$ spaces.

Definition. An $L^{p}(X ; E)$ multiplier of the semigroup $\exp (-t L)$ is a function $b$ belonging to $L_{\mathrm{loc}}^{1}(0, \infty)$ for which the strong operator limit of Bochner integrals

$$
M(b) \eta=\lim _{\varepsilon \rightarrow 0+, T \rightarrow \infty} \int_{\varepsilon}^{T} b(t) \exp (-t L) \eta \mathrm{d} t \quad\left(\eta \in L^{p}(X ; E)\right)
$$

exists and defines a bounded linear operator on $L^{p}(X ; E)$.

We wish to give sufficient conditions on $b$ that it define an $L^{p}(X ; E)$ multiplier. In order to make progress, it is necessary to impose geometrical conditions on the Banach space $E$.

Definition. A Banach space $E$ is said to be a $U M D$ space if there is a constant $C_{E}$ for which

$$
\int\left\|\sum_{n} a_{n} d_{n}(\omega)\right\|_{E}^{2} \mathrm{~d} \omega \leqq C_{E}^{2} \int\left\|\sum_{n} d_{n}(\omega)\right\|_{E}^{2} \mathrm{~d} \omega
$$

for all transforms of finite martingale difference sequences $\left(d_{n}\right)$ with values in $E$ by constants $a_{n}$ with $\left|a_{n}\right| \leqq 1$.

Burkholder and Bourgain showed that $E$ is a $U M D$ space if and only if the Hilbert transform is bounded on $L^{p}(\mathbf{R} ; E)$ for $1<p<\infty$. See [1, Theorem 2.7]. For a discussion of examples and a spectral theory of groups of operators on $U M D$ spaces we refer the reader to [1]. Here we simply record that $L^{q}(\mathbf{R} ; \mathbf{C})$ is a $U M D$ space for $1<q<\infty$ but not for $q=1$ nor $q=\infty$.

Our multiplier theorems are proved in Sections 3 and 4 for $U M D$ spaces using the group $L^{-i u}$ of imaginary powers of $L$. Under technical conditions stated below, the multipliers for the semigroup $e^{-t L}$ and the group $L^{-i u}$ are related by

$$
\int_{0}^{\infty} b(t) e^{-t L} \mathrm{~d} t=\frac{1}{2 \pi i} \int_{c-i \infty}^{c+i \infty} \Gamma(-s) B(s) L^{s} \mathrm{~d} s \quad(c<0)
$$

where $B(s)$ is the Mellin transform of $t b(t)$.

\section{Transference method}

Our method of proving that multipliers are bounded on $L^{p}(X ; E)$ is to take a 
multiplier theorem for the group of translation operators on the real line and transfer it to a group of operators on $L^{p}(X ; E)$. We begin by presenting a transference principle to deal with locally bounded groups of operators. See [1, Theorems $4.1,5.6]$ for the case of uniformly bounded $C_{0}$ groups of operators.

Definition. Let $u \mapsto T_{u}$ be a strongly continuous representation of the real line as a $C_{0}$ group of bounded linear operators on a Banach space $E$. Then the operator norms $\left\|T_{s}\right\|_{E}$ are uniformly bounded on compact subsets of $\mathbf{R}$ by $[4, \mathrm{p} .304]$. We define the modular function of $T$ to be

$$
\tau(u)=\sup \left\{\left\|T_{s}\right\|_{E}:|s| \leqq|u|\right\} \quad(u \in \mathbf{R})
$$

By an application of the uniform boundedness theorem given in $[4, p .306]$, the function $\tau(u)$ is at most of exponential growth in $|u|$.

Theorem 2.1. Let a be an integrable function supported on the interval $[-u, u]$. Then the operator

$$
T(a): g \mapsto \int_{-\infty}^{\infty} a(s) T_{s} g \mathrm{~d} s \quad(g \in E)
$$

has operator norm at most $2^{1 / p} \tau^{3}(u)\|\Lambda(a)\|_{L^{P}(R ; E)}$ where $\|\Lambda(a)\|_{L^{p}(R ; E)}$ is the norm of the convolution operator

$$
\Lambda(a): f \mapsto \int_{-\infty}^{\infty} a(v-s) f(v) \mathrm{d} v \quad\left(f \in L^{p}(\mathbf{R} ; E)\right)
$$

on $L^{P}(\mathbf{R} ; E)$ for $1 \leqq p<\infty$.

Proof. Let $h$ be an element of $E$. We observe that for $|s| \leqq u$

$$
\|h\|_{E} \leqq\left\|T_{-s}\right\|_{E}\left\|T_{s} h\right\|_{E} \leqq \tau(u)\left\|T_{s} h\right\|_{E}
$$

Hence setting $h=T(a) g$ we have $\|T(a) g\|_{E}^{p} \leqq \tau(u)^{p}\left\|T_{s} T(a) g\right\| p$. We integrate from $s=-u$ to $s=u$ to get

$$
2 u\|T(a) g\|_{E}^{p} \subseteq \tau(u)^{p} \int_{-u}^{u}\left\|T_{s} T(a) g\right\|_{E}^{p} \mathrm{~d} s
$$


We introduce the vector-valued function $k(t)=\aleph_{[-2 u, 2 u]}(t) T_{t} g$ where $\aleph_{[-2 u, 2 u]}$ is the indicator function of $[-2 u, 2 u]$. For $|s| \leqq u$ we have

$$
\left\|T_{s} T(a) g\right\|_{E}^{p}=\left\|\int_{-\infty}^{\infty} a(t-s) k(t) \mathrm{d} t\right\|_{E}^{p}=\|\Lambda(a) k(s)\|_{E} .
$$

Hence we can estimate the integral in the previous expression (9) by

$$
\int_{-u}^{u}\left\|T_{s} T(a) g\right\|_{E}^{p} \mathrm{~d} s \leqq\|\Lambda(a)\|_{L^{P}(R ; E)}^{p}\|k\|_{L^{p}(R ; E)}^{p} .
$$

Now we estimate $\|k(t)\|_{E}$ pointwise to give

$$
\int_{-\infty}^{\infty}\|k(t)\|_{E}^{p} \mathrm{~d} t \leqq \int_{-2 u}^{2 u} \sup _{|s| \leqq 2 u}\left\|T_{s} g\right\|_{E}^{p} \mathrm{~d} t \leqq 4 u \tau(2 u)^{p}\|g\|_{E}^{p} .
$$

Combining (11) and (12) with (9) we obtain

$$
\|T(a) g\|_{E}^{p} \leqq 2 \tau(u)^{p} \tau(2 u)^{p}\|\Lambda(a)\|_{L^{p}(R ; E)}^{p}\|g\|_{E}^{p}
$$

Since $\tau(u)$ is at most of exponential growth in $u$ we have that $\tau(2 u) \leqq \tau(u)^{2}$. Taking $p^{\text {th }}$ roots of (13), we obtain the stated result.

\section{Multipliers for semigroups}

Let us suppose that $L$ satisfies the Hypotheses 1.1 (i)-(iv). Then we can define a $C_{0}$ unitary group of operators $L^{-i u}(u \in \mathbb{R})$ on $L^{2}(X ; \mathbf{C})$ by the functional calculus of the self-adjoint operator $L$. Hence we can define a family of operators $T_{u}=L^{-i u} \otimes I$ on $\mathscr{C} \otimes I$. If $T_{u}$ extends to define a strongly continuous group of operators on $L^{p}(X ; E)$, then we can use the transference techniques of the previous section to construct multipliers. We will use the Mellin transform to convert transferred convolution operators (7) involving $L^{-i u}$ into multipliers (3) involving $e^{-i L}$. We will write $L^{-i u}$ for $L^{-i u} \otimes I$ and similarly abbreviate the other notation.

Definition. Let $b$ be a function for which $t^{-\sigma} b(t) \in L^{1}(0, \infty)$ for $\sigma_{1}<\sigma<\sigma_{2}$ where $0 \leqq \sigma_{1}$. We define the Mellin transform of $t b(t)$ by

$$
B(s)=\int_{0}^{\infty} x b(x) x^{s-1} \mathrm{~d} x
$$

Lemma 3.1. (i) The function $B(s)$ is analytic in the strip $-\sigma_{2}<\Re s<-\sigma_{1}$. 
Suppose further that

(ii) $B(-\sigma+i u) \in L_{u}^{1}(-\infty, \infty)$ for $\sigma \in\left(\sigma_{1}, \sigma_{2}\right)$ and

(iii) $B(-\sigma+i u) \rightarrow 0$ uniformly as $|u| \rightarrow \infty$ in the strip $\sigma_{1}+\varepsilon<\sigma<\sigma_{2}-\varepsilon$ for each $\varepsilon>0$. Then for $\sigma_{1}<\sigma<\sigma_{2}$ the following identity holds

$$
\int_{0}^{\infty} b(t) e^{-t \lambda} \mathrm{d} t=\frac{1}{2 \pi} \int_{-\infty}^{\infty} a_{\sigma}(u) \lambda^{-\sigma-i u} \mathrm{~d} u \quad(\lambda>0)
$$

where $a_{\sigma}(u)=\Gamma(\sigma+i u) B(-\sigma-i u)$.

Proof. The statement (i) follows from Morera's Theorem and the Dominated Convergence Theorem.

The conditions (i), (ii) and (iii) constitute the hypotheses of the Mellin Inversion Theorem of $[6$, p. 273$]$ so we can write

$$
t b(t)=\frac{1}{2 \pi i} \int_{c-i \infty}^{c+i \infty} t^{-s} B(s) \mathrm{d} s \quad\left(t>0,-\sigma_{2}<c<-\sigma_{1}\right)
$$

where the line of integration $\mathfrak{R} s=c=-\sigma$ lies in the strip where $B(s)$ is holomorphic. We multiply this identity (16) by $t^{-1} e^{-t \lambda}$ and integrate with respect to $t$ over $(0, \infty)$. When $\lambda>0$ the integrals converge absolutely and we can change the order of integration to obtain

$$
\begin{aligned}
\int_{0}^{\infty} b(t) e^{-t \lambda} \mathrm{d} t= & \frac{1}{2 \pi i} \int_{c-i \infty}^{c+i \infty} \int_{0}^{\infty} t^{-s-1} e^{-i \lambda} \mathrm{d} t B(s) \mathrm{d} s \\
& =\frac{1}{2 \pi i} \int_{c-i \infty}^{c+i \infty} \lambda^{s} \Gamma(-s) B(s) \mathrm{d} s \quad\left(\lambda>0,-\sigma_{2}<c<-\sigma_{1}\right) .
\end{aligned}
$$

Remark. If $b \in L^{1}(0, \infty)$ then the Mellin transform $B(s)$ of $t b(t)$ is bounded on the line $\Re s=0$. It follows from the Riemann-Lebesgue Lemma that $B(i u) \rightarrow 0$ as $|u| \rightarrow \infty$. To ensure condition (ii) is satisfied one replaces $b$ by

$$
b_{k}(x)=k^{2} \int_{0}^{1} b(x / t) t^{k-2} \log \frac{1}{t} \mathrm{~d} t
$$

By the arguments presented in [6, p. 276] the Mellin transform of $t b_{k}(t)$ is $(k /(k+s))^{2} B(s)$. This converges boundedly to $B(s)$ as $k \rightarrow \infty$.

Definition. We introduce a norm

$$
\|h\|_{\mathcal{M}}=\sup \left\{|h(t)|+\left|t h^{\prime}(t)\right|+\left|t^{2} h^{\prime \prime}(t)\right|: t \in \mathbf{R}\right\}
$$


on the space of twice continuously differentiable functions on the real line.

For $j \geqq 1$ we introduce the modified de la Vallée Poussin kernels

$$
v_{j}(t)=\frac{\exp \left(i 3.2^{j-1} t\right)}{2 \pi}\left(\frac{\sin ^{2} 2^{j-1} t}{2^{j-1} t^{2}}-\frac{\sin ^{2} 2^{j-2} t}{2^{j-3} t^{2}}\right)
$$

The Fourier transforms $\hat{v}_{j}(t)$ are supported in $\left[2^{j-1}, 5.2^{j-1}\right]$ and $\hat{v}_{j}(t)=1$ for $t \in\left[2^{j}, 2^{j+1}\right]$. For $j \leqq-1$ we set $v_{j}(t)=v_{-j}(-t)$ and introduce $v_{0}$ so that the sequence $\left(\hat{v}_{j}\right)_{j=-\infty}^{\infty}$ gives a partition of unity of the real line.

Theorem 3.2. Suppose that $L^{-i n}$ defines a strongly continuous group of operators on $L^{p}(X ; E)$ where $E$ is a $U M D$ space and $1<p<\infty$. Suppose that $B$ satisfies (i), (ii) and (iii) of Lemma 3.1 with $\sigma_{1}=0$ and that there is $K<\infty$ for which

$$
\sum_{j=-\infty}^{\infty} \tau\left(2^{|j|}\right)^{9}\left\|v_{j} * h_{\sigma}\right\|_{\mathcal{A}} \leqq K \quad\left(0<\sigma<\sigma_{2}\right)
$$

where

$$
h_{\sigma}(v)=e^{-\sigma v} \int_{0}^{\infty} \exp \left(-x e^{-v}\right) b(x) \mathrm{d} x \quad(\sigma>0, v \in \mathbf{R})
$$

Then $M(b)$ defines a bounded linear operation on $L^{p}(X ; E)$.

Proof. By the assumptions on $L$ and the equation (15) of Lemma 3.1 we can write

$$
\int_{0}^{\infty} b(t) e^{-t L} \eta \mathrm{d} t=\frac{1}{2 \pi} \int_{-\infty}^{\infty} a_{o}(u) L^{-\sigma-i u} \eta \mathrm{d} u \quad\left(\eta \in \mathscr{C} \otimes E, \sigma_{1}<\sigma<\sigma_{2}\right)
$$

as an identity of strongly convergent integrals. This is an immediate consequence of the Fubini-Tonelli Theorem since the spectral measure $P(\mathrm{~d} \lambda)$ is strongly countably additive.

We now calculate the Fourier transform of $a_{\sigma}(u)$. We express its defining identity as a double integral

$$
\begin{aligned}
a_{\sigma}(u) & =\int_{0}^{\infty} t^{\sigma+i u-1} e^{-t} \mathrm{~d} t \times \int_{0}^{\infty} b(x) x^{-\sigma-i u} \mathrm{~d} x \\
& =\int_{0}^{\infty} \int_{0}^{\infty} t^{\sigma+i u-1} x^{-\sigma-i u} e^{-t} \mathrm{~d} t b(x) \mathrm{d} x
\end{aligned}
$$

We use the transformation $t=x e^{v}$ to express the inner integral in (20) as an integral over the real line, so that 


$$
a_{\sigma}(u)=\int_{0}^{\infty} \int_{-\infty}^{\infty} e^{i u v} e^{\sigma v} \exp \left(-x e^{v}\right) \mathrm{d} v b(x) \mathrm{d} x .
$$

Since the integrals in (20) converge absolutely we can change the order of integration here to write

$$
a_{\sigma}(u)=\int_{-\infty}^{\infty} e^{i u v} \int_{0}^{\infty} e^{\sigma v} \exp \left(-x e^{v}\right) b(x) \mathrm{d} x \mathrm{~d} v
$$

By the Fourier Inversion Theorem the Fourier transform of $a_{\sigma}$ is given by

$$
\hat{a}_{\sigma}(-v)=2 \pi \int_{0}^{\infty} e^{-\sigma v} \exp \left(-x e^{-v}\right) b(x) \mathrm{d} x=2 \pi h_{\sigma}(v) .
$$

We shall prove that under the stated hypotheses the family of operators

$$
S_{\sigma, N}=\sum_{j=-N}^{N} \int_{-\infty}^{\infty} \hat{v}_{j}(u) a_{\sigma}(u) L^{-i u} \mathrm{~d} u .
$$

is bounded on $L^{p}(X ; E)$ with a bound independent of $N$ as $\sigma \rightarrow 0+$. This suffices to give the stated result as the following approximation argument shows. Let

$$
T_{\sigma, N}=\sum_{j=-N}^{N} \int_{-\infty}^{\infty} \hat{v}_{j}(u) L^{-\sigma-i u} \mathrm{~d} u .
$$

Suppose that $\left\|S_{\sigma, N}\right\|_{L^{p}(X ; E)} \leqq C$ for $0<\sigma<\sigma_{2}$ and $N \geqq 1$. For $\eta \in \mathscr{C} \otimes E$ we can write

$$
T_{\sigma, N} \eta=S_{\sigma, N} \eta+S_{\sigma, N}\left(L^{-\sigma} \eta-\eta\right) .
$$

Given $\varepsilon>0$ we can choose $\delta>0$ for which $\left\|L^{-\sigma} \eta-\eta\right\|_{L P(X ; E)} \leqq \varepsilon$ if $0<\sigma<\delta$ so that

$$
\begin{gathered}
\left\|T_{\sigma, N} \eta\right\|_{L^{P(X ; E)}} \leqq\left\|S_{\sigma, N} \eta\right\|_{L^{P}(X ; E)}+\left\|S_{\sigma, N}\right\|_{L^{P}(X ; E)}\left\|L^{-\sigma} \eta-\eta\right\|_{L^{P(X ; E)}} \\
\leqq C\|\eta\|_{L^{P(X ; E)}}+C \varepsilon .
\end{gathered}
$$

Letting $N \rightarrow \infty$ we conclude from the integral representation (19) that $\|M(b) \eta\|_{L^{p}(X ; E)} \leqq$ $C\left\|_{\eta}\right\|_{L^{P}\left(X_{i} E\right)}$. Since $\mathscr{C} \otimes E$ is dense in $L^{p}(X ; E)$ this gives the desired estimate on $M(b)$.

The main idea behind the proof is that we can use the transference principle to estimate the operator norm of each summand of (23). By Theorem 2.1 we have

$$
\left\|\int_{-\infty}^{\infty} \hat{v}_{j}(u) a_{\sigma}(u) L^{-i u} \mathrm{~d} u\right\|_{L P(X ; E)} \leqq 2^{1 / p} \tau\left(5.2^{|j|-1}\right)^{3}\left\|\Lambda\left(\hat{v}_{j} a_{\sigma}\right)\right\|_{L P(R ; L P(X ; E))}
$$


since $\hat{v}_{j}$ is supported in $\left[-5.2^{|j|-1}, 5.2^{|j|-1}\right]$.

The Banach space $L^{p}(X ; E)$ is also a $U M D$ space and so the vector-valued version of the Hörmander-Mihlin Theorem from [5, 1.1] may be applied. We obtain that

$$
\left\|\Lambda\left(\hat{v}_{j} a_{\sigma}\right)\right\|_{L^{p}\left(R: L^{P}(X ; E)\right)} \leqq C_{p, E}\left\|v_{j} * h_{\sigma}\right\|_{\mathcal{M}} .
$$

Combining (26) with the previous expressions (25) and (23) gives us the bound

$$
\left\|S_{\sigma, N}\right\|_{L^{p}(X ; E)} \leqq \sum_{j=-\infty}^{\infty} C_{p, E} \tau\left(5.2^{|j|-1}\right)^{3}\left\|v_{j} * h_{\sigma}\right\|_{\mathcal{M}} \leqq C_{p, E} K,
$$

where the final inequality follows from (18) since $5.2^{|j|-1} \leqq 3.2^{|j|}$.

\section{Homomorphic multipliers}

For convenience we recall Stirling's Formula $[8,4.42]$. For any fixed value of $x$

$$
\Gamma(x+i y) \asymp e^{-\pi|y| / 2}|y|^{x-1 / 2} \sqrt{(2 \pi)} \quad(|y| \rightarrow \infty) .
$$

Theorem 4.1. Suppose that $L^{-i u}$ defines a $C_{0}$ group of operators on $L^{p}(X ; E)$ where $E$ is a UMD space and $1<p<\infty$. Suppose further that there is $\psi$ with $0 \leqq \psi<\pi$ for which

(i) $\tau(u) \leqq C_{1} \exp \left(\left(\frac{\pi}{2}+\psi\right)|u|\right)$ for some $C_{1}<\infty$ and all real $u$.

(ii) The function $b$ is bounded and holomorphic in the cone

$$
K_{\psi+\varepsilon}=\{z:|z|>0,|\arg (z)|<\psi+\varepsilon\}
$$

for some $\varepsilon>0$ with $\psi+\varepsilon<\pi$.

(iii) There is $C_{2}(\varepsilon)<\infty$ for which $|b(z)| \leqq C_{2}|z|^{-2}$ for $z \in K_{\psi+\varepsilon}$.

Then $M(b)$ is bounded on $L^{p}(X ; E)$.

Proof. We shall show that the right-hand side of (19) defines a bounded operator on $L^{p}(X ; E)$. Recall that $a_{\sigma}(u)=\Gamma(\sigma+i u) B(-\sigma-i u)$. We begin by recording some facts about the Mellin transform $B(s)$ of $t b(t)$. By conditions (ii) and (iii) of the Theorem the function $B(s)$ is holomorphic on the strip $-1<\Re s<1$. In particular it is holomorphic near to the axis $R s=0$. We can estimate the decay of $B(i u)$ as $u \rightarrow \infty$ by turning the line of integration in (14) to the line $\arg t=\phi$ where $\phi=\psi+\varepsilon / 2$. This line lies in $K_{\psi+\varepsilon}$. We obtain the estimate

$$
\begin{aligned}
|B(\sigma+i u)| & =\left|\int_{0}^{\infty}\left(v e^{i \phi}\right)^{\sigma+i u} b\left(v e^{i \phi}\right) e^{i \phi} \mathrm{d} v\right| \\
& \leqq e^{-\phi u} \int_{0}^{\infty} v^{\sigma}\left|b\left(v e^{i \phi}\right)\right| \mathrm{d} v \leqq C_{3}(\sigma, \varepsilon) e^{-\phi u} \quad(u>0,-1<\sigma<1) .
\end{aligned}
$$


A corresponding result holds for $u<0$.

In general $B(-s) \Gamma(s)$ will have a simple pole at $s=0$. By Cauchy's Theorem the integral in (19) along $\mathfrak{R} s=-\sigma$ may be replaced by an integral along the line $\Re s=0$ with an indentation about $s=0$. By spectral theory we see that as the radius of the indentation decreases to zero, the integral about the indentation tends to $2^{-1} B(0) \eta$ for each $\eta \in \mathscr{C} \otimes E$. The integral along $\Re s=0$ may be treated as a Cauchy principal value integral with singularity at $s=0$. We take $s=-i u$ and split this integral into a sum of integrals corresponding to the ranges of integration $u \in[-1,1]$ and $|u|>1$ respectively. Let $\rho$ be a smooth bump function identically one on $[-1,1]$ and supported in $[-2,2]$. We write $a_{0}(u)=\rho(u) a_{0}(u)+(1-\rho(u)) a_{0}(u)$ and consider the small values of $|u|$ first.

We use the Laurent expansion of $\Gamma(s)$ about $s=0$ to write the Cauchy Principal Value Integral as

$\mathrm{PV} \int_{-2}^{2} \rho(u) a_{0}(u) L^{-i u} \eta \mathrm{d} u=\mathrm{PV} \int_{-2}^{2}-B(0) \rho(u) L^{-i u} \eta \frac{\mathrm{d} u}{u}+\int_{-2}^{2} f(u) L^{-i u} \eta \mathrm{d} u \quad(\eta \in \mathscr{C} \otimes E)$

where $f$ is continuous on $[-2,2]$. Clearly the last term in (31) defines a bounded operator on $L^{p}(X ; E)$.

The convolution operator $\Lambda\left(u^{-1} \rho(u)\right)$ is bounded on $L^{p}\left(\mathbf{R} ; L^{p}(X ; E)\right)$ by the Hörmander-Mihlin Theorem of [5, Theorem 1.1, Remark 3.2] since $L^{p}(X ; E)$ is a $U M D$ space. It follows from Theorem 2.1 that the first summand in (31) defines a bounded operator on $L^{p}(X ; E)$. (We can recognise this operator as a transferred version of the finite Hilbert transform. See [1, Corollary 2.18] and [6, p. 467].)

The part of the integral (19) corresponding to large values of $|u|$ is absolutely convergent. We use the triangle inequality and definition of $\tau$ to obtain

$$
\left\|\int_{|| u \mid>1\}} B(-i u) \Gamma(i u) L^{-i u} \eta \mathrm{d} u\right\|_{L^{p}(X ; E)} \leqq \int_{\{|u|>1]}|B(-i u) \Gamma(i u)| \tau(|u|) \mathrm{d} u \times\|\eta\|_{L^{p}(X ; E)} .
$$

By Stirling's formula (28) combined with (30) and the hypothesis (i) this is

$$
\leqq \int_{[|u|>1]} C_{3} e^{-\phi|u|} e^{-\pi|u| / 2} C_{1} \exp \left(\left(\frac{\pi}{2}+\psi\right)|u|\right) \mathrm{d} u \times\|\eta\|_{L^{p}(X ; E)} \leqq C_{4}(\varepsilon)\|\eta\|_{L^{p}(X: E)}
$$

Corollary 4.2. Suppose that $L^{-i u}$ defines a $C_{0}$ group of operators on $L^{p}(X ; E)$ where $E$ is a UMD space and $1<p<\infty$. Suppose further that there is $\psi$ with $0<\psi<\pi / 2$ and $C_{1}<\infty$ for which $(i) \tau(u) \leqq C_{1} \exp \left(\left(\frac{\pi}{2}-\psi\right)|u|\right)$ for each real $u$. Then the cone $\{w: \Re w>0$, $|\arg (w)|<\psi\}$ is contained in the resolvent set of $L$, regarded as an operator in $L^{p}(X ; E)$. Further, $(-L)$ generates a holomorphic semigroup on $L^{p}(X ; E)$, bounded in each cone $K_{\psi-\varepsilon}=\{z: \Re z>0,|\arg (z)|<\psi-\varepsilon\}$ for $\varepsilon>0$.

Proof. By the Hille-Yoshida Theorem 12.3.1 of [4] a necessary and sufficient 
condition for $(-L)$ to generate a holomorphic semigroup $e^{-z L}$ with $\left\|e^{-z L}\right\|_{L^{p}(X ; E)} \leqq M_{\varepsilon}$ for $z$ in $K_{\psi-\varepsilon}$ is that the integer powers of the resolvent satisfy $\left\|w^{m}(w+L)^{-m}\right\|_{L^{p}(X ; E)} \leqq M_{\varepsilon}$ for all $w \in K_{\psi-\varepsilon}$ and $m \geqq 1$. We obtain a formula for powers of the resolvent by setting $b(t)=t^{m-1} e^{-w t}$ in (19). Using familiar identities satisfied by the Gamma function we get

$$
\begin{gathered}
\Gamma(-s) B(s)=w^{-(m+s)} \Gamma(-s) \Gamma(m+s) \\
=-w^{-(m+s)}(m+s-1)(m+s-2) \ldots(s+1) \pi \operatorname{cosec}(\pi s) .
\end{gathered}
$$

Hence by (19) we have

$$
\frac{w^{m}}{(w+L)^{m}}=\int_{c-i \infty}^{c+i \infty}-\frac{(m-1+s)(m-2+s) \ldots(1+s)}{2 \pi i w^{s} \Gamma(m)} \pi \operatorname{cosec}(\pi s) L^{s} \mathrm{~d} s(c<0)
$$

as an identity of operators on $\mathscr{C} \otimes E$.

We estimate the right hand side of (34) by the technique of the proof of Theorem 4.1. The line of integration in (34) is replaced by a curve consisting of the imaginary axis $\sigma=0$ with an indentation about $\sigma+i u=0$. We require to estimate the integrand of (34) on $\mathfrak{R} s=0$. For real values of $u$ we have

$$
\left|\frac{(m-1-i u)(m-2-i u) \ldots(1-i u)}{\Gamma(m)}\right|^{2}=\prod_{j=1}^{m-1}\left(1+\frac{u^{2}}{j^{2}}\right)
$$

Considering the product formula for the sine function we see that (35) is

$$
\leqq \frac{\sinh \pi u}{\pi u} \leqq e^{\pi u} \quad(u \geqq 1)
$$

We use this to estimate (34) by

$$
\begin{aligned}
& \left\|\int_{[|u|>1]} \frac{(m-1-i u)(m-2-i u) \ldots(1-i u)}{2 \Gamma(m)} w^{i u} \operatorname{cosec}(\pi i u) L^{-i u} \mathrm{~d} u\right\|_{L^{P}(X ; E)} \\
& \leqq \int_{1}^{\infty} 2 \exp \left(\frac{\pi}{2} u+|\arg (w)| u\right) \tau(u) e^{-\pi u} \mathrm{~d} u \leqq 2 \int_{1}^{\infty} \exp \left(\left(\psi-\varepsilon-\frac{\pi}{2}\right) u\right) \tau(u) \mathrm{d} u .
\end{aligned}
$$

Using the assumption (i) on $\tau$ we see that this latest integral is bounded by $C_{1} \varepsilon^{-1}$.

Using the same argument as with (31) above, one can show that the part of the integral (34) corresponding to $\{|u|<1\}$ is bounded with a bound independent of $m$ and $w$ by comparing it with the transferred finite Hilbert transform. Hence the operators $w^{m}(w+L)^{-m}$ extend to define a uniformly bounded family of operators on $L^{p}(X ; E)$ for $w \in K_{\psi-\varepsilon}$ and $m \geqq 1$. 
Remark. The formula (34) with $m=1$ is equivalent to a Mellin transform formula given by Sneddon $[6$, p. 521].

\section{Examples}

Example 5.1. Let $\Delta$ be the classical Laplace operator on the real line and $E$ be any $U M D$ space. It is known that $(-\Delta)^{i u} \otimes I$ defines a $C_{0}$ group of operators on $L^{p}(\mathbf{R} ; E)$ for $1<p<\infty$. The modular function $\tau(u)$ is of polynomial growth in this case [6, Theorem 1.1]. Conversely, if $(-\Delta)^{i n} \otimes I$ is a locally bounded group of operators on $L^{p}(\mathbf{R} ; E)$ for some $p$ with $1<p<\infty$ then $E$ is a $U M D$ space [3, p. 402].

Example 5.2. The conditions (ii) and (iii) of Hypotheses 1.1 are satisfied when $L$ is the generator of a symmetric diffusion semigroup $\exp (-t L)$. We take $X$ to be a smooth complete manifold and suppose that in local co-ordinates $L$ has the shape

$$
L f=-e(x)^{-1} \sum_{j, k=1}^{n} \frac{\partial}{\partial x_{j}}\left(a_{j k}(x) \frac{\partial}{\partial x_{k}} f\right)-c(x) f \quad\left(f \in C_{c}^{\infty}(X)\right)
$$

with $\left[a_{j k}(x)\right]$ positive definite, $e(x)>0$ and $c(x) \leqq 0$. Under general conditions on the coefficients the closure of $(-L) \mid C_{c}^{\infty}(X ; \mathrm{C})$ generates a $C_{0}$ contraction semigroup on $L^{p}(X ; C)$ for $1 \leqq p \leqq \infty$. See [2, p. 412], [7, p. 66]. The Poisson semigroup, which is also a contraction semigroup on $L^{p}(X ; \mathrm{C})$ for $1 \leqq p \leqq \infty$, may be obtained by subordination. The integer powers $L^{m}(m \geqq 1)$ are essentially self-adjoint on $C_{c}^{\infty}(X ; \mathbf{C})$. The condition (i) of 1.1 does not generally hold when $X$ is compact since the spectrum is discrete and $L$ need not be invertible. In this case one considers semigroups defined on the orthogonal complement of the zero eigenspace of $L$.

It follows from Stein's multiplier theorem for symmetric diffusion semigroups [6, $\mathrm{p}$. 121] that the imaginary powers $L^{i u}$ of $L$ are bounded on $L^{p}(X ; \mathbf{C})$ for $1<p<\infty$ with

$$
\left\|L^{i u}\right\|_{L^{p}(X ; C)} \leqq C_{p}|u|^{-1 / 2} e^{\pi|u|}
$$

for large values of $|u|$.

Acknowledgements. I should like to thank Dr S. Guerre-Delabriere, Dr D. Kershaw and Dr T. A. Gillespie for helpful conversations.

Note added in proof. After this paper had been submitted Dr Guerre-Delabriere informed the author that a result similar to Corollary 4.2 appears on p. 437 of J. PRÜSS and H. SOHR, On operators with bounded imaginary powers in Banach spaces, Math. Z. 203 (1990), 427-452. 


\section{REFERENCES}

1. E. Berkson, T. A. Gillespie and P. S. Muhly, Abstract spectral decompositions guaranteed by the Hilbert transform, Proc. London Math. Soc. (3) 53 (1986), 489-517.

2. P. R. Chernoff, Essential self-adjointness of powers of generators of hyperbolic equations, $J$. Funct. Anal. 12 (1973), 401-414.

3. S. Guerre-Delabriere, Some remarks on complex powers of $(-\Delta)$ and UMD spaces, Illinois J. Math. 35 (1991), 401-407.

4. E. Hille and R. S. Phillips, Functional analysis and semi-groups (American Math. Soc., Colloquium Publications, Vol. XXXI, Providence, R.I., 1957).

5. T. R. McConNell, On Fourier multiplier transformations of Banach-valued functions, Trans. Amer. Math. Soc. 285 (1984), 739-757.

6. I. N. SNEDdon, The use of integral transforms (McGraw-Hill, New York, 1972).

7. E. M. STEIN, Topics in harmonic analysis related to the Littlewood Paley Theory (Annals of Math. Studies 63, Princeton, New Jersey, 1970).

8. E. C. Titchmarsh, The theory of functions, second edition (OUP, Oxford, 1938).

Department of Mathematics and Statistics

LANCASTER UNIVERSITY

LANCASTER LA1 4YF

ENGLAND 